\title{
Abatacept versus other biologics in methotrexate inadequate responders with rheumatoid arthritis: you like tomato and I like tomahto... let's call the whole thing off
}

\author{
Yusuf Yazici* \\ See related research by Guyot et al., http://arthritis-research.com/content/13/6/R204
}

\begin{abstract}
In the absence of head-to-head trials, analysis of available data from randomized clinical trials allows for comparison of the efficacy of biologic agents for the treatment of rheumatoid arthritis. Methotrexate (MTX) inadequate responder trials provide data suggesting no major differences among any of the biologic agents, which is also confirmed in MTX naive population trials, when available, possibly a more reliable comparison group. The decision to pick one over the other should focus on safety, long-term survival of the drug and ease of use, which is for the most part influenced by patient preferences.
\end{abstract}

In a previous issue of Arthritis Research \& Therapy, Patricia Guyot and colleagues present their elegant paper of a meta-analysis comparing abatacept to other biologics in rheumatoid arthritis (RA) patients who had an inadequate response (IR) to methotrexate (MTX) [1]. The authors compare MTX IR studies to see if various biologic agents differ in achieving improvement in function as measured by the Health Assessment Questionnaire, ACR50 and DAS28 $<2.6$ responses, all robust measures of improvement. In their analysis, patients had similar responses with abatacept compared to other biologics etanercept, adalimumab, infliximab, certolizumab, rituximab and tocilizumab - at 24 and 52 weeks. As discussed in the paper, differences of methodology and types of patients enrolled in each individual trial may account for the few differences reported among the biologics. In my

*Correspondence: yusuf.yazici@nyumc.org

NYU Hospital for Joint Diseases, New York, NY 10003, USA opinion, these are not clinically relevant and I agree with the conclusion reached by the authors that similar responses should be expected when these biologics are used for the treatment of RA patients who are MTX-IR.

Currently most of the available biologic agents have MTX-IR indications, as these are the patients where a biologic agent would be most commonly used. However, 'MTX-IR' is poorly defined in clinical trials, where it is up to the treating physician to decide what an inadequate response' is. Hence, depending on when and where the study was conducted, different types of patients, with different histories of MTX use, dosage and duration, all considered MTX-IR, may be included, and this makes head-to-head comparison difficult. Commonly the disease activity levels are similar at baseline but what really happened to them while on MTX prior to enrollment is not clear. A better comparison would probably be if patients with early RA who are MTX-naive were compared, as these patients would be more likely to be similar in their previous RA treatment and hence lead to more robust comparisons. We previously carried out such a study using number needed to treat as a comparator and got similar results, confirming the findings of the current study [2]. In addition, there are data to suggest that, in the real world routine care setting, there is no difference in time to response and the response achieved over one year among abatacept, adalimumab, etanercept and infliximab treated RA patients [3].

This brings up the question of whether we need headto-head clinical trials, which are usually expensive and, because of the similar efficacy demonstrated in various analyses, likely need large numbers of patients to be able to show superiority. This has led to several of the currently ongoing studies to use non-inferiority for efficacy outcomes as the main study aim. I think the current report adds to the already available literature that, as far as efficacy is concerned, there is very little 
difference among the current biologics. As most patients seen in routine care would not be able to fulfill criteria to be included $[4,5]$ as they generally have milder disease, any difference that may exist in analyses of randomized clinical trials may not be reflected in the real world. Most patients in the real world have less disease burden where any biologic would likely work with the same effectiveness. In addition, patients with comorbid conditions are excluded from randomized clinical trials and this makes it even harder to guess at the efficacy of any given biologic agent when used in routine care.

In summary, the available data, be it in MTX-IR or early RA, MTX-naive patients, suggests that the biologic agents currently used for the treatment of RA seem to have similar efficacy. There are currently no biomarkers, and there may never be any that would be useful and predictive in individual patients in routine clinical care, to help us distinguish among biologics. The decision to pick one over the other should focus on safety, long-term survival of the drug and ease of use, which is, for the most part, influenced by patient preferences. The main determinant of outcome depends not on which agent is used but when it is used and how aggressively disease activity is monitored and treated with a focus on reaching low disease activity or remission, with whichever composite index we chose to use as our target [6]. Use of outcome measures shows some regional variation among and within countries, especially if access to biologics is dependent on having a certain level of disease severity, yet overall we still have a long way to go until measurement is part of routine care. It is time to stop picking favorites among the biologic agents and focus on how we can get more physicians to monitor RA outcomes that would allow us to be as aggressive as RA demands us to be.

\section{Abbreviations}

$I R$, inadequate response; MTX, methotrexate; RA, rheumatoid arthritis.

\section{Competing interests}

YY has acted as a consultant and/or received research support from Abbott, BMS, Celgene, Genentech, Merck, Pfizer and UCB.

Published: 21 February 2012

\section{References}

1. Guyot $P$, Taylor P, Christensen R, Pericleous L, Poncet C, Lebmeier M, Drost P, Bergman G: Abatacept with methotrexate versus other biologic agents in treatment of patients with active rheumatoid arthritis despite methotrexate: a network meta-analysis. Arthritis Res Ther 2011 13:R204

2. Yazici Y, Swearingen CJ, Nadkarni A, Rosenblatt L: Comparative efficacy and tolerability of biologic therapies in early rheumatoid arthritis utilizing a Bayesian approach [abstract]. Arthritis Rheum 2011, 63(Suppl S10):S484.

3. Yazici Y, Filopoulos M, Swearingen CJ: Comparative effectiveness and time to response among adalimumab, abatacept, etanercept and infliximab for the treatment of rheumatoid arthritis in a real world routine care registry. Arthritis Rheum 2011, 63(Suppl):S873.

4. Sokka T, Pincus T: Most patients receiving routine care for rheumatoid arthritis in 2001 did not meet inclusion criteria for most recent clinical trials or american college of rheumatology criteria for remission. J Rheumatol 2003, 30:1138-1146.

5. Gogus F, Yazici Y, Yazici H: Inclusion criteria as widely used for rheumatoid arthritis clinical trials: patient eligibility in a Turkish cohort. Clin Exp Rheumatol 2005, 23:681-684.

6. Yazici Y: Treatment of rheumatoid arthritis: we are getting there. Lancet 2009, 374:210-211.

doi:10.1186/ar3688

Cite this article as: Yazici Y: Abatacept versus other biologics in methotrexate inadequate responders with rheumatoid arthritis: you like tomato and I like tomahto... let's call the whole thing off. Arthritis Research \& Therapy 2012, 14:104. 